\title{
New Odd Generalized Exponential - Exponential Distribution: Its Properties and Application
}

\author{
Badamasi Abba and Singh VV* \\ Department of Mathematics, Yusuf Maitama Sule University, Nigeria
}

Submission: January 29, 2018; Published: April 25, 2018

*Corresponding author: Singh VV, Department of Mathematics, Yusuf Maitama Sule University, Kano, Nigeria, Email: singh_vijayvir@yahoo.com

\section{Abstract}

In academia, inducing a shape parameter to an exponential probability model has been an old practice and quite number of approaches for doing so were discussed in the literature. In this article, we introduce two shape parameters to the exponential probability model using an idea proposed in a recent study and this resulted in a new three-parameter probability distribution entitled New Odd Generalized ExponentialExponential distribution. The proposed probability model can serve as a better alternative to a sister probability distribution entitled Odd Generalized Exponential-Exponential distribution developed in an earlier research using a certain probability generator. Different properties of the proposed probability model comprising moment, moment generating function, quantile function, survival function and hazard function were obtained. For illustrative purposes, two datasets were analyzed to compare the performance of our proposed probability distribution with the existing odd generalized exponential-exponential distribution; and it was observed that, the proposed model fits the two datasets better.

Keywords : Generalized exponential distribution; Moments; Quantile function; Parameter estimation

Abbreviations : GE: Generalized Exponential; OGE-W: OGE-Weibull; OGE: Odd Generalized Exponential; OGE-Fr: OGE-Fréchet; OGE-N: OGENormal; NOGE-E: New Odd Generalized Exponential-Exponential

\section{Introduction}

Inducing of new shape parameter(s) to an existing probability model expands the model into a larger family of distributions and this provides a significantly skewed and heavy-tails probability distribution models. The extended or generalized model can provide better flexibility in modeling lifetime data for better actualization of phenomenon contained in the dataset. An approach to the construction of flexible parametric models is to insert appropriate competing models into a larger model by adding shape parameter(s) [1]. This has opened rooms for many researches in the distribution theory. The induction of parameter(s) has been proved useful in exploring tail properties and also for improving the goodness-of-fit of the proposed generator family [2]. The exponential distribution is one of the most important distribution with wide range of applications in statistical practice. It has been identified as a life testing model among many other applications. Attempts to increase the flexibility of the exponential distribution gave rise to many generalized family of distribution [3]. Several generalizations of exponential distribution have been studied, among them are generalized exponential distributions [4], ExponentialWeibull distribution, The Kumaraswamy exponential-Weibull distribution and many more have appeared in literature. The cdf and pdf of the exponential distribution are given by

$$
G(x)=1-e^{-\lambda x}
$$

And

$$
g(x)=\lambda e^{-\lambda x}
$$

Respectively, where $\lambda>0$ and $x>0$ and $\lambda$ is the scale parameter.

In 2015, Tahir et al. [5] have developed a new class of univariate distributions called the odd generalized exponential (OGE) family and studied each of the OGE-Fréchet (OGE-Fr) distribution, the OGE-Weibull (OGE-W) distribution and the OGE-Normal (OGE-N) distribution. This method is flexible because the hazard rate shapes could be increasing, decreasing, bathtub and upside-down bathtub. In this article, we defined a new three parameter probability model, called new odd generalized exponential-exponential (NOGE-E) distribution using the new family of univariate distributions introduced by Tahir et al. [5] as an alternative to two parameters OGEED developed by Maiti \& Pramanik [6]. The main feature of this model is that two additional parameters are introduced in the subject distribution which provides greater flexibility in the form of new distributions. A random variable $\mathrm{X}$ is said to have generalized exponential (GE) distribution with parameters if the cumulative distribution function (cdf) is given by 


$$
F(x)=\left(1-e^{-\alpha x}\right)^{\beta}, \quad x>0, \alpha>0, \beta>0
$$

The odd generalized exponential family suggested by Tahir et al. [5] is defined as follows. If $G(x ; \psi)$ is the CDF of any distribution and $\bar{G}(x ; \psi)=1-G(x ; \psi)$ is the survival function, then the $O G E-X$ is defined by replacing in CDF of GE in equation (1) by $\frac{G(x ; \psi)}{\bar{G}(x ; \psi)}$ to get the cdf of the new family of distribution as follows:

$$
F(x ; \alpha, \beta, \vartheta)=\left(1-e^{-\alpha \frac{G(x ; y)}{G(x ; y)}}\right)^{\beta}, x>0, \alpha>0, \beta>0, \psi>0
$$

And the probability density function of the family of distributions is

$f(x ; \alpha, \beta, \zeta)=\frac{\alpha \beta g(x ; \psi)}{(1-G(x ; \psi))^{2}} e^{-\alpha \frac{G(x ; \psi)}{\bar{G}(x ; \psi)}}\left(1-e^{-\alpha \frac{G(x ; \psi)}{\bar{G}(x ; \psi)}}\right)^{\beta}(1.5)$

Where $\alpha$ and $\beta$ are the generator additional parameters to be added to any baseline probability distribution and, $\psi$ is the parameter vector of the baseline distribution.

This article is outlined as follows. In Section 2, we define the cumulative distribution, density, reliability and hazard functions of the new odd generalized exponential-exponential(NOGE-E) distribution respectively. We introduce the statistical properties including the limiting behavior, the quantile function, the median, the moments, the moment generating function, the mode and the Renyi entropy of the new distribution are provided in section 3. Section 4 presents the order statistics. Also, maximum likelihood estimation of the parameters is determined in Section 5. Finally, some applications of NOGE-E distribution using two lifetime data is presented in Section 6.

Definition 1.1 A random variable $X$ is said to have a New Odd Generalized Exponential- Exponential distribution, if its probability density function (pdf) has the form

$$
f(x ; \alpha, \beta, \lambda)=\alpha \beta \lambda e^{\lambda x} e^{-\alpha\left(e^{\lambda x}-1\right)}\left(1-e^{-\alpha\left(e^{\lambda x}-1\right)}\right)^{\beta-1}
$$

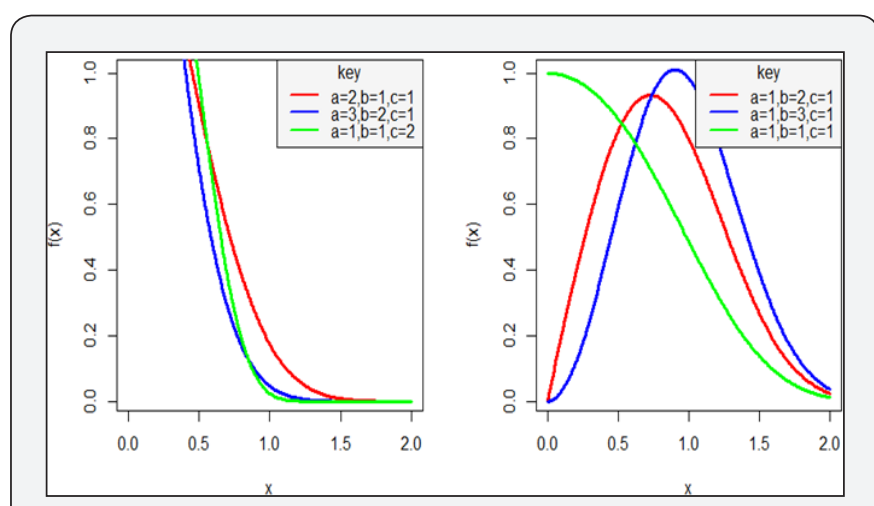

Figure 1: Plots of the pdf for some values of the parameters.

The plots of the density of NOGE-E distribution are displayed in Figure 1 for various values of the parameters.

The cumulative distribution function (cdf) of the NOGE-E distribution is

$$
F(x ; \alpha, \beta, \lambda)=\left(1-e^{-\alpha\left(e^{2 x-1}\right)}\right)^{\beta}, \quad x>0, \alpha>0, \beta>0, \lambda>0
$$

The cumulative distribution plot of NOGE-E distribution is displayed in Figure 2 for various values of the parameters.

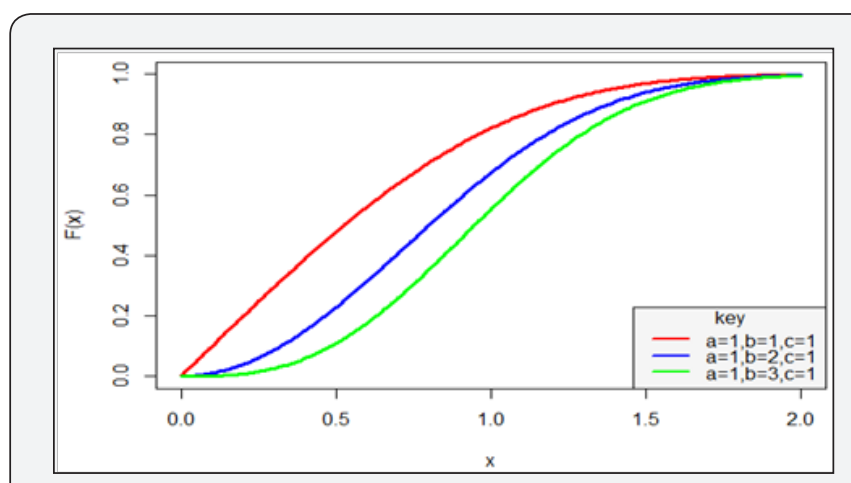

Figure 2: Plot of the cdf for some values of the parameters.

Let be a random variable with pdf and cdf given in Equations (1.6) and (1.7) respectively, we write $X \sim N O G E-E(\psi)$, where the vector $\psi$ is defined by $\psi=(\alpha, \beta, \lambda)$. As a result of (1.6) and (1.7), the reliability and hazard functions of the distribution are given as

$$
S(x)=1-F(x)=1-\left(1-e^{-\alpha\left(e^{\lambda x}-1\right)}\right)^{\beta}
$$

And

$$
H(x)=\frac{f(x)}{S(x)}=\frac{\alpha \beta \lambda e^{\lambda x} e^{-\alpha\left(e^{2 x}-1\right)}\left(1-e^{-\alpha\left(e^{2 x}-1\right)}\right)^{\beta-1}}{1-\left(1-e^{-\alpha\left(e^{2 x}-1\right)}\right)^{\beta}}
$$

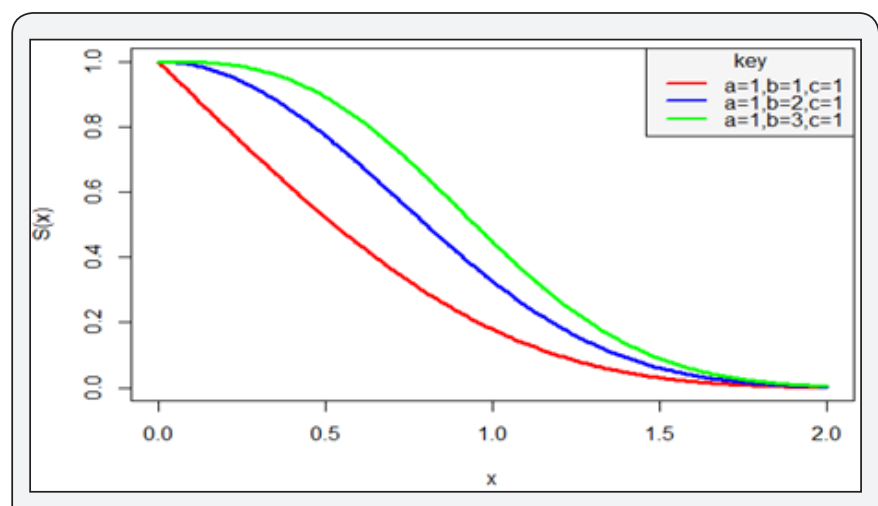

Figure 3: Plot of the cdf for some values of the parameters.

The reliability and hazard rate plot of NOGE-E distribution is displayed in Figure $3 \& 4$ for various values of the parameters.

\section{Properties of the distribution}

Useful expansion of the density and distribution functions: The useful expansion of the cdf and pdf of the $N O G E-E(\psi)$ distribution using a well-known generalized binomial and power series expansion are presented below. It 
is provided to motivate the analytical deviation of some basic properties of the distribution. The generalized binomial and power series expansion are given as

$$
(1-z)^{b-1}=\sum_{i=0}^{\infty}\left(\begin{array}{c}
b-1 \\
i
\end{array}\right)(-1)^{i} z^{i}=\sum_{i=0}^{\infty} \frac{\tilde{\mathrm{A}}(b)(-1)^{i} z^{i} \text { for }}{\tilde{\mathrm{A}}(b-i)}|z|<1 \text { and } b>0
$$

And

$$
e^{-x}=\sum_{j=0}^{\infty} \frac{(-1)^{j} x^{j}}{j !}
$$

Then, for $\alpha, \beta, \lambda \in \mathbb{R}$ the pdf of the NOGE-E( $\psi)$ distribution can be expressed as

$f(x ; \alpha, \beta, \lambda)=\beta \lambda \sum_{i=0}^{\infty} \sum_{j=0}^{\infty} \sum_{k=0}^{\infty}\left(\begin{array}{l}j \\ k\end{array}\right)\left(\begin{array}{c}\beta-1 \\ i\end{array}\right) \frac{(-1)^{i+j+k} \alpha^{j+1}(1+i)^{j}}{j !} e^{-(k-j-1) \lambda x}$

The cdf of the NOGE-E( $\psi)$ distribution can be expressed as

$$
F(x ; \alpha, \beta, \lambda)=\sum_{i=0}^{\infty}\left(\begin{array}{c}
\beta-1 \\
i
\end{array}\right)(-1)^{i} e^{-i \alpha\left(e^{\lambda x}-1\right)}
$$

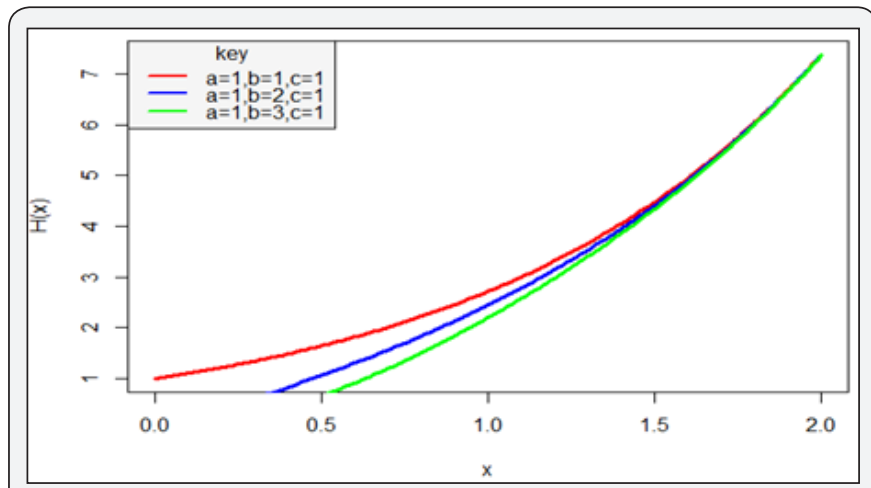

Figure 4: Plot of the Hazard rate function for some values of the parameters.

\section{Quantile function and random number generation}

Quantiles are used for estimation and simulation of distribution parameters. By inverting equation (1.7), the quantile function of the $N O G E-E(\psi)$ distribution is $x(q)=\ln \left(1-\frac{\ln \left(1-q^{1 / \beta}\right)}{\alpha}\right)^{\frac{1}{2}}$, for $0<q<1$ (2.3)

In particular, the median of the distribution is

$$
x(0.5)=\ln \left(1-\frac{\ln (1-[0.5]}{\alpha} \text { for } 0<q<1\right.
$$

To simulate a random variable from the NOGE-E $(\psi)$ distribution. Let $q$ be a uniform random variable on the interval $(0,1)$ then it follows that $X(q) \sim N O G E-E(\psi)$ distribution.

\section{Quantile based skewness and kurtosis}

Skewness measures the degree of long-tail while kurtosis measures the degree of tail heaviness. Based on the quantile function $X(q)$,the Bowley measure of skewness and Moor's measure of kurtosis are

$$
\begin{aligned}
& S k=\frac{X(3 / 4)-2 X(1 / 2)+X(1 / 4)}{X(3 / 4)-X(1 / 4)} \\
& K=\frac{X(7 / 8)-X(5 / 8)+X(3 / 8)+X(1 / 8)}{X(6 / 8)-X(2 / 8)}
\end{aligned}
$$

The quantile based skewness and kurtosis are of advantage over the corresponding classical measures, this is because, they can be computed even when the classical moment does not exist [7].

\section{Moment}

Let $X$ be a random variable which follows $N O G E-E(\psi)$ distribution, then the moment of the distribution is as stated in theorem 1 below.

Theorem 1: If is a random variable distributed according to $N O G E-E(\psi)$ distribution in equation (1.6). Then, the $\mathrm{r}^{\text {th }}$ moment of the distribution is given by

$$
\mu_{r}^{\prime}=\sum_{i=0}^{\infty} \sum_{j=0}^{\infty} \sum_{k=0}^{\infty} \frac{(-1)^{i+j+k} \alpha^{j+1}(1+i)^{j} \Gamma(\beta+1) \Gamma(r+1)}{\lambda^{r}(k-j-1)^{r+1}(\beta-i-1) !(j-k) ! i ! k !}
$$

Proof: by the definition of the moment

$$
\mu_{r}^{\prime}=E\left(X^{r}\right)=\int_{0}^{\infty} x^{r} f(x) d x
$$

Applying equation (2.3) into (2.6), after some little algebraic manipulations, we arrived at the moments of the NOGE-E distribution as

$$
\mu_{r}^{\prime}=\sum_{i=0}^{\infty} \sum_{j=0}^{\infty} \sum_{k=0}^{\infty} \frac{\left.\left.(-\tilde{A})^{j+j+k} \mathrm{~b}\right) \tilde{\mathrm{A}}(1+1)\right)^{j} \quad \beta+\quad r+}{\lambda^{r}(k-j-1)^{r+1}(\beta-i-1) !(j-k) ! i ! k !}
$$

This complete the proof.

\section{Moment generating function}

Let $X$ be a random variable which follows $N O G E-E(\psi)$ distribution, then the moment generating function $M_{x}(\theta)$, of the distribution is as stated in theorem 2 below.

Theorem 2: If is a random variable distributed according to $N O G E-E(\psi)$ distribution in equation (1.6). Then, the moment generating function of the distribution is given by

$$
M_{x}(\theta)=\sum_{i=0}^{\infty} \sum_{j=0}^{\infty} \sum_{k=0}^{\infty} \sum_{m=0}^{\infty} \frac{\left.(-1)^{i+j+k} \alpha^{j+1}(\tilde{\mathbb{A}}(t))^{j} \Gamma\right)(\beta+1) \quad m+\theta^{m}}{\lambda^{m}(k-j-1)^{m+1}(\beta-i-1) !(j-k) ! i ! k ! m !}
$$

Proof: by definition of the moment generating function

$$
M_{x}(\theta)=E\left(e^{\theta X}\right)=\int_{0}^{\infty} e^{\theta x} f(x) d x(16)
$$

applying series expansion on $e^{\theta x}$, equation (16) can be written as

$$
M_{x}(\theta)=\sum_{m=0}^{\infty} \frac{\theta^{m}}{m !} E\left(X^{m}\right)
$$


Since $E\left(X^{m}\right)=E\left(X^{r}\right)$ with $(m=r)$, by making use of equation (2.7) we obtain the moment generating function of the $N O G E-E(\psi)$ distribution as

$M_{x}(\theta)=\sum_{i=0}^{\infty} \sum_{j=0}^{\infty} \sum_{k=0}^{\infty} \sum_{m=0}^{\infty} \frac{(-1)^{i+j+k} \alpha^{j+1}(1+i)^{j} \Gamma(\beta+1) \Gamma(m+1) \theta^{m}}{\lambda^{m}(k-j-1)^{m+1}(\beta-i-1) !(j-k) ! i ! k ! m !}$
This completes the proof.

\section{Mode}

By differentiating the pdf of the distribution with respect as

$$
f^{\prime}(x)=f(x)\left[\alpha \beta \lambda e^{\lambda x} e^{-\alpha\left(e^{2 x}-1\right)}\left((\beta-1)\left[1-e^{-\alpha\left(e^{\lambda x}-1\right)}\right]^{-1}-1\right)+1\right](2.10)
$$

and since $f(x)>0$, the mode is the solution of the equation with respect to $x$

$$
\alpha \beta \lambda e^{\lambda x} e^{-\alpha\left(e^{2 x}-1\right)}\left[1-e^{-\alpha\left(e^{2 x-1}\right)}\right]^{\beta-1} \times\left[\alpha \beta \lambda e^{\lambda x} e^{-\alpha\left(e^{2 x-1}\right)}\left((\beta-1)\left[1-e^{-\alpha\left(e^{2 x-1}\right)}\right]^{-1}-1\right)+1\right]=0
$$

Equation (2.11) can be solved numerically using Mathematica package

\section{Renyi entropy}

The Renyi entropy of a random variable represent the measure of randomness or variation of uncertainty of a system. It is defined as

$$
I_{R}(\gamma)=\frac{1}{1-\gamma} \log \left(\int_{-\infty}^{\infty} f^{\gamma}(x) d x\right), \gamma>0 \text { and } \gamma \neq 1
$$
by

Theorem 3: The Renyi entropy for the distribution is given

$$
I_{R}(\gamma)=\frac{\gamma}{1-\gamma} \log \beta+\frac{\gamma-1}{1-\gamma} \log \lambda \alpha+\frac{1}{1-\gamma} \log \left(\sum_{i, j}^{\infty}\left(\begin{array}{c}
\gamma-1 \\
i
\end{array}\right)\left(\begin{array}{c}
\gamma(\beta-1) \\
j
\end{array}\right) \frac{(-1)^{i+j} \Gamma(j+1)}{(\gamma+j)^{j+1}}\right)(3
$$

Proof: Since

$$
\int_{-\infty}^{\infty} f^{\gamma}(x) d x=(\alpha \beta \lambda)^{\gamma} \int_{0}^{\infty} e^{\gamma \lambda x} e^{-\gamma \alpha\left(e^{\lambda x}-1\right)}\left(1-e^{-\alpha\left(e^{\lambda x}-1\right)}\right)^{\gamma(\beta-1)} d x
$$

Then, by making use of $y=e^{-\alpha\left(e^{e x-}-1\right)}$ it follows that

$$
\int_{-\infty}^{\infty} f^{\gamma}(x) d x=(\alpha \lambda)^{\gamma-1} \beta^{\gamma} \int_{0}^{1}\left(1-\frac{\log y}{\alpha}\right)^{\gamma-1} y^{\gamma-1}(1-y)^{\gamma(\beta-1)} d y
$$

Expanding the binomial terms in equation (3.4), lead to the desired proof.

\section{Estimation with inference}

In this section, the method of maximum likelihood is considered to estimate the distribution unknown parameters. Let $x_{1}, \ldots x_{n}$ be a sample of size from the NOGE $-E(\psi)$ distribution with parameter vector Then the log-likelihood function for $\psi$ is given by;

$$
\begin{aligned}
& l(\psi)=\log L\left(x_{1}, \cdots x_{n} / \psi\right)=\log \prod_{i=1}^{n} f\left(x_{i} / \psi\right)=n \ln \alpha+n \ln \beta+n \ln \lambda-\lambda \sum_{i=1}^{n} x_{i}-\alpha \\
& \times \sum_{i=1}^{n}\left(e^{\lambda x_{i}}-1\right)+(\beta-1) \sum_{i=1}^{n} \ln \left(1-e^{-\alpha\left(e^{\lambda x_{i}}-1\right)}\right)
\end{aligned}
$$

Differentiating (21) with respect to each of the parameters in $\psi$ we obtain

$$
\begin{aligned}
& \frac{\partial l}{\partial \mathrm{a}}=\frac{n}{\alpha}-\sum_{i=1}^{n}\left(e^{\lambda x_{i}}-1\right)+(\beta-1) \sum_{i=1}^{n} \frac{e^{\lambda x_{i}}-1}{\left(e^{\alpha\left(e^{\lambda x_{i}}-1\right)}-1\right)} \\
& \frac{\partial l}{\partial \hat{\mathrm{a}}}=\frac{n}{\beta}+\sum_{i=1}^{n} \ln \left(1-e^{-\alpha\left(e^{\lambda x_{i}}-1\right)}\right)(23) \\
& \frac{\partial l}{\partial \ddot{\mathrm{e}}}=\frac{n}{\lambda}+\sum_{i=1}^{n} x_{i}-\alpha \sum_{i=1}^{n} x_{i} e^{\lambda x_{i}}+\alpha(\beta-1) \sum_{i=1}^{n} \frac{x_{i} e^{\lambda x_{i}}}{\left(e^{\alpha\left(e^{2 \lambda-}-1\right)}-1\right)}
\end{aligned}
$$

The maximum likelihood estimators (MLEs) $\hat{\psi}=(\hat{\alpha}, \hat{\beta}, \hat{\lambda})$ of the $\psi=(\alpha, \beta, \lambda)^{\prime}$, can be determined by solving the three above systems of non-linear equations numerically for $\psi=(\alpha, \beta, \lambda)^{\prime}$, This is achieved using the Newton-Raphson maximization method in R package [8]. Real data application

Table 1: Data set I.

$3.70,2.74,2.73,2.50,3.60,3.11,3.27,2.87,1.47,3.11,3.56,4.42$, $2.41,3.19,3.22,1.69,3.28,3.09$,

$1.87,3.15,4.90,1.57,2.67,2.93,3.22,3.39,2.81,4.20,3.33,2.55$, $3.31,3.31,2.85,1.25,4.38,1.84$,

$0.39,3.68,2.48,0.85,1.61,2.79,4.70,2.03,1.89,2.88,2.82,2.05,3.65$, $3.75,2.43,2.95,2.97,3.39$,

$2.96,2.35,2.55,2.59,2.03,1.61,2.12,3.15,1.08,2.56,1.80,2.53$

Table 2: Data set II.

$6.545,9.289,7.543,6.956,6.492,5.459,8.120,4.706,8.687,2.997$, 8.591, 6.129, 11.038, 5.381,

$6.958,4.288,6.522,4.137,7.459,7.495,6.573,6.538,5.589,6.087$, $5.807,6.725,8.532,6.663$

$6.369,7.024,8.336,9.218,7.945,6.869,6.352,4.700,6.948,9.254$, $5.009,7.489,7.398,6.033$

$10.092,7.496,4.531,7.974,8.799,7.683,7.224,7.365,6.923,5.640$ $5.434,7.937,6.515,6.476$,

$6.071,10.491,5.923$ 
Table 3: Parameter Estimates for Data set I.

\begin{tabular}{|c|c|}
\hline The model & MLE's of the parameter(s) \\
\hline $\operatorname{OGEED}(\lambda, \alpha)$ & $\hat{\lambda}=1.07068, \hat{\alpha}=0.03482$ \\
\hline $\operatorname{NOGEED}(\lambda, \alpha, \beta)$ & $\hat{\lambda}=.6707, \hat{\alpha}=.2454, \hat{\beta}=2.2940$ \\
\hline
\end{tabular}

To compare the performance of the NOGE-E( $\psi)$ distribution with the existing odd generalized exponentialexponential distribution based on a set of goodness-of-fit tests, we used two (2) real data sets. The first data set given in Table 1 , shows the breaking stress of carbon fibres (in $\mathrm{Gba}$ ) reported by Cordeiro and Lemonte and the second data set is from an accelerated life test of 59 conductors. The data have been previously used by Nasir et al. [9] and Oguntunde et al. [10] (Table 1-6).

Table 4: Performance comparisons.

\begin{tabular}{|c|c|c|}
\hline The model & Log-likelihood & AIC \\
\hline $\operatorname{OGEED}(\lambda, \alpha)$ & -88.08835 & 180.1767 \\
\hline $\operatorname{NOGEED}(\lambda, \alpha, \beta)$ & -85.68582 & 177.3716 \\
\hline
\end{tabular}

Table 5: Parameter Estimates for Data set II.

\begin{tabular}{|c|c|}
\hline The model & MLE's of the parameter(s) \\
\hline $\operatorname{OGEED}(\lambda, \alpha)$ & $\hat{\lambda}=.59447, \hat{\alpha}=.01035$ \\
\hline $\operatorname{NOGEED}(\lambda, \alpha, \beta)$ & $\hat{\lambda}=.2149, \hat{\alpha}=.74284, \hat{\beta}=8.2303$ \\
\hline
\end{tabular}

Table 6: Performance comparisons.

\begin{tabular}{|c|c|c|}
\hline The Model & Log-likelihood & AIC \\
\hline $\operatorname{OGEED}(\lambda, \alpha)$ & -116.3297 & 236.6595 \\
\hline $\operatorname{NOGEED}(\lambda, \alpha, \beta)$ & -109.8608 & 225.7215 \\
\hline
\end{tabular}

\section{Conclusion}

In this article, the new odd generalized exponentialexponential (NOGE-E) distribution is introduced by applying the OGE family of distribution proposed by Tahir et al. [5]. Some of the statistical properties of the distribution have been derived [11,12]. The maximum likelihood estimation is used to estimate the parameters of the distribution. Two data sets have been employed to compare its performance with existing two parameter model and the superiority of the developed distribution has been established based on the data sets used. This confirmed the claims of higher flexibility of the new distribution by inducing new shape parameter.

\section{References}

1. Aryal GR, Tsokos CP (2009) On the Transmuted Extreme Value Distribution with Application. Nonlinear Analysis Theory Method and Application 71: 1401-1407.

2. Saboor A, Kamal M, Ahmad M (215) The Transmuted ExponentialWeibull Distribution with Application. Pak J Statist 31(2): 229-250.

3. Oguntunde PE, Adejumo AO (2015) Transmuted Inverse-Exponential distribution. International Journal of Advanced Statistics and Probability 3(1): 1-7.

4. Gupta RD, Kundu D. Generalized Exponential Distribution Austral. N Z J Statist 41(2): 173-188.

5. Tahir MH, Cordeiro GM, Alizadeh M, Mansoor M (2015) The Odd Generalized family of Distributions with Applications. Journal of Statistical Distributions and Applications 2:1.

6. Maiti SS, Pramanik S (2015) Odd Generalized Exponential-Exponential Distribution. International Journal of Mathematics and Statistics Invention 13: 2321-4767.

7. Bowley AL (1920) Element of Statistics. PS King.

8. RényiA (1961) On measures of entropy and information. Proc. 4th Berkeley Symp Math Stat Probab, Univ California Press, Berkeley, pp. 547-561.

9. Nasiri PI, Makhdoom, Yaghoubian B (2011) Estimation parameters of the weighted exponential distribution. Aust J Basic Applied Sci 1: 4.

10. Oguntunde PE, Owoloko EA, Balogun OS (2016) On A New Weighted Exponential distribution: Theory and Application. Asian Journal of Applied Sciences 9(1): 1-12.

11. Moors JJ (1988) A quantile alternative for kurtosis. JR Stat Soc Ser D 37: $25-32$.

12. Chiwa Musa Dalah, Singh VV (2017) A Survey and Assessment Report of Hiv/Aids Awareness of in North-Eastern Nigeria. International Journals of Advanced Research in Computer Science and Software Engineering 7(11): 105-112. 

(C) C) This work is licensed under Creative

DOI: 10.19080/BBOAJ.2018.05.555686

\section{Your next submission with Juniper Publishers} will reach you the below assets

- Quality Editorial service

- Swift Peer Review

- Reprints availability

- E-prints Service

- Manuscript Podcast for convenient understanding

- Global attainment for your research

- Manuscript accessibility in different formats

( Pdf, E-pub, Full Text, Audio)

- Unceasing customer service

Track the below URL for one-step submission https://juniperpublishers.com/online-submission.php 